\title{
Adaptable beam profiles from a dual-cavity Nd:YAG laser
}

\author{
D. J. KIM, ${ }^{1}$ J. I. MACKENZIE, ${ }^{2}$ AND J. W. KIM ${ }^{1, *}$ \\ ${ }^{1}$ Department of Applied Physics, Hanyang University ERICA, Ansan, Gyeonggi-do 426-791, Republic of Korea \\ ${ }^{2}$ Optoelectronics Research Center, University of Southampton, Southampton, SO17 1BJ, UK \\ *Corresponding author: jwk7417@hanyang.ac.kr
}

Received XX Month XXXX; revised XX Month, XXXX; accepted XX Month XXXX; posted XX Month XXXX (Doc. ID XXXXX); published XX Month XXXX

\begin{abstract}
We report a technique to tailor a laser beam profile from a donut to quasi-top-hat intensity distribution, directly from the laser, simply achieved by simultaneous excitation and control of the relative contributions of the fundamental (TEM ${ }_{00}$ ) and first order Laguerre-Gaussian (LGo1) transverse modes. Exploiting a dual-cavity configuration with a single Nd:YAG gain element, adaptable continuous-wave laser beam profiles from the primary cavity could be obtained by varying the diffraction loss of an acousto-optic modulator in the secondary cavity. We investigated the resultant beam profiles as a function of pump power and the AOM diffraction loss, and discuss the prospects for tunable laser beams profiles. (C) 2016 Optical Society of America
\end{abstract}

OCIS codes: (140.0140) Lasers and laser optics; (140.3300) Laser beam shaping; (140.3410) Laser resonators; (140.3530) Lasers, neodymium.

http://dx.doi.org/10.1364/OL.99.099999

Diode-end-pumped solid-state lasers have matured to such a level that they are now serving numerous applications in areas such as industry, defense, medicine and spectroscopy due to low lasing thresholds and high optical efficiencies. One of the primary attractions of this laser architecture is the ability to selectively excite one or a few transverse modes of the laser cavity by matching the pumping light distribution in the gain medium to the targeted laser modes [1]. It is becoming clear that tailored laser beam profiles are playing a major role in materials processing applications, as the intensity distribution at the work piece dictates the resultant pattern of the induced damaged zone, and the effects on the zone surrounding it. Moreover, optimizing the energy distribution can lead to dramatic improvements in the efficiency and speed of the ablation, hence processing time [2]. For example, it is well known that a fundamental Gaussian beam $\left(\mathrm{TEM}_{00}\right)$ has an effective intensity twice that of an equivalent sized top-hat beam, leading to damage in a central region and possibly wasted energy, in terms of materials processing in the wings. Similarly damage to optical components sets the limit for energy/power extraction from lasers due to the higher on axis intensity. Increasing the number of transverse modes provides a means of increasing the energy content of the beam, however, non-uniform amplification of each discrete mode (or multi- mode seed beams in amplifiers) causes deformation of the transverse intensity profile and mode competition, triggering dynamic instabilities in the intensity distribution and output power $[3,4]$. For this reason, there has been significant effort devoted to controlling the transverse beam profile of lasers, to optimize the conditions for various applications, particularly precision micro-machining.

Several techniques have been employed to generate bespoke laser beam profiles. Passive methods based on geometric beam transforming optics, such as specially designed fiber waveguides [5, 6], mirror/lens arrays [7, 8] and spatial light modulators [9], are straightforward to implement and widely used, but each of these methods suffers from a lack of flexibility due to a one-to-one correspondence in their transformation function. Alternatively, tailored beam profiles can be generated directly in the laser cavity using intra-cavity beam shaping elements [10], or a graded-phase output coupler that encourages simultaneous lasing of multiple transverse modes [11, 12]. This approach has the advantage of simplicity in configuration and the prospect of high efficiency, but it is difficult to precisely control the distribution of power between modes due to saturation of inversion, thermo-optical effects, and mode competition. Pump-beam shaping techniques, that is, tailoring the pump beam profile to excite targeted laser modes, has been shown to be effective in overcoming this difficulty, however, this does require complex pumping systems such as a capillary-fiber $[13,14]$.

Here we report a new strategy for directly generating a laser beam with a tailored transverse intensity distribution from a solid-state laser based on a dual-cavity configuration incorporating a tunable loss component, namely an acousto-optic modulator (AOM). The control of the AOM diffraction loss allows us to precisely define the gain distribution in the laser medium, thus producing a laser beam with the targeted intensity profile. For demonstration, we have applied this approach to a diode-end-pumped Nd:YAG laser yielding hundreds of milliwatts of laser output with tailored beam profiles determined by the controllable diffraction loss of the AOM.

The Nd:YAG dual-cavity laser configuration used in our experiments is shown in Fig. 1. The laser employs a coupled dual-cavity configuration, i.e. the primary and the secondary cavities share a common gain medium and pump-in-coupling mirror, then separate through a polarization beam splitter to two linear cavities, as described in more detail in Ref. 15. To achieve a low-loss polarization beam splitter, we have employed an antireflection (AR)-coated calcite beam displacer along with an edge mirror providing independent spatial 
paths for the two polarized cavity modes in the respective cavities. Thus the output from the primary cavity was linearly polarized regardless of the transverse mode content. A 1.0 at.\% Nd:YAG rod with a $5 \mathrm{~mm}$ length was used as the gain medium and mounted in a watercooled aluminum heat-sink maintained at $19{ }^{\circ} \mathrm{C}$ positioned in close proximity to the pump in-coupling mirror. The pump beam was provided by a high power diode laser at $808 \mathrm{~nm}$ coupled to a typical step-index 0.22NA multimode fiber with a $200 \mu \mathrm{m}$ core diameter. The pump absorption efficiency in the Nd:YAG rod was measured to be $\sim 81 \%$ under optimum operating conditions.

The primary cavity comprised a plane pump input coupler (IC) with high reflectivity $(>99.8 \%)$ at the lasing wavelength $(\sim 1 \mu \mathrm{m})$ and high transmission $(>95 \%)$ at the pump wavelength $(808 \mathrm{~nm})$, a planoconvex lens of $75 \mathrm{~mm}$ focal length AR-coated at the lasing wavelength, and a plane output coupler with $20 \%$ transmission at the lasing wavelength (OC1). In order to excite both $\mathrm{TEM}_{00}$ and $\mathrm{LG}_{01}$ modes simultaneously, the pump beam was focused to have a waist radius of $\sim 220 \mu \mathrm{m}$ in the crystal since a calculated TEM 00 waist was $\sim 170 \mu \mathrm{m}$ [16].

The secondary cavity included the shared pump input coupler (IC), and a second AR-coated lens of a $75 \mathrm{~mm}$ focal length and a plane output coupler with $5 \%$ transmission at the lasing wavelength (OC2). The secondary cavity also had a similar $\mathrm{TEM}_{00}$ mode size in the gain medium compared to the primary cavity. Both cavities were carefully designed such that the TEMon mode size in the Nd:YAG rod was insensitive to the inherent thermal lensing. An aperture (AP) was inserted in the secondary cavity to suppress higher order modes, especially $\mathrm{LG}_{01}$ mode in this experiment. Furthermore, we placed the AOM in the secondary cavity to provide a tunable cavity loss for the TEMoo mode, still resulting in a lower lasing threshold than for the primary cavity. The round-trip loss of each cavity for the fundamental mode, including diffraction losses, was determined by the Findlay-Clay method [17]. When the AOM was inactive, the round-trip loss was determined to be $4.5 \%$ for the primary cavity and $9.0 \%$ for the secondary cavity (without the influence of the aperture, i.e. AP was fully opened). Higher cavity loss for the secondary cavity is due to the additional edge mirror and the insertion loss of the AOM. When AP was partially closed to ensure operation of the secondary cavity on the TEMo0 mode only, the round-trip loss increased to $14.8 \%$, however, the total cavity loss for the secondary cavity including the transmission of the output coupler, $19.8 \%$, was still lower than that for the primary cavity, $24.5 \%$.

We monitored the near-field beam profile of the output from the primary cavity at a relayed image-plane of the OC1, using a silicon CCD camera (Spiricon BS-USB-SP620). For an AOM loss of $0 \%$, only the $\mathrm{LG}_{01}$ mode with the donut intensity profile was generated in the primary cavity as expected [15]. This is due to the overlapped gain

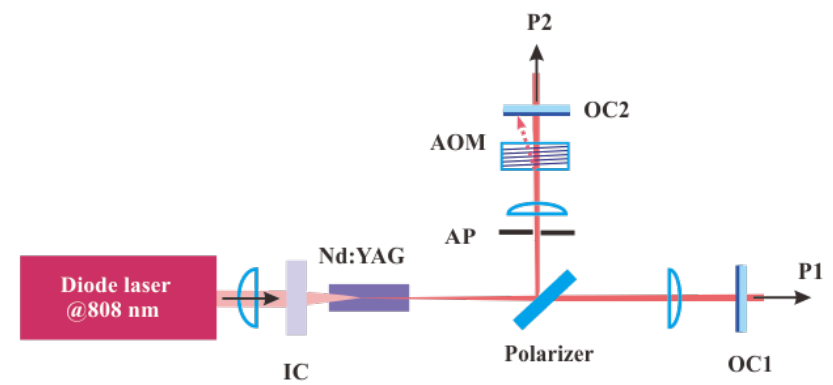

Fig. 1. Schematic diagram of the Nd:YAG dual-cavity laser. IC: input coupler, OC: output coupler, AP: aperture, AOM: acoustooptic modulator distribution in the common Nd:YAG crystal because the total cavity loss of the primary cavity for the TEM ${ }_{00}$ mode, including the transmission loss of the output coupler, is higher than in the secondary cavity. Therefore the average population inversion in the center of the pumped region is pinned at the lower gain-level threshold set by the lowest cavity losses. Figure 2 shows the measured transverse intensity distributions of the laser output (P1) from the primary cavity, at an incident pump power of $3.2 \mathrm{~W}$, and corresponding additional AOM diffraction round-trip losses of $0 \%, 0.5 \%, 1.5 \%$ and $2.5 \%$ in the secondary cavity, that is, round-trip losses in that cavity of $14.8 \%$, $15.3 \%, 16.3 \%$ and $17.3 \%$, respectively. Corresponding output powers were $143 \mathrm{~mW}, 184 \mathrm{~mW}, 230 \mathrm{~mW}$ and $239 \mathrm{~mW}$, respectively, due to the increasing TEMo0 mode contribution to P1 associated with the increasing round-trip loss, and reducing P2 output, for the secondary cavity. Higher AOM losses effectively isolated the secondary cavity, i.e. it did not reach threshold, which resulted in multimode laser operation of the primary cavity. These results clearly show that the relative contribution of the $\mathrm{TEM}_{00}$ mode to the total output from the primary cavity was determined by the threshold-gain state of the secondary cavity. Consequently, sufficient gain is made available for the TEM 00 mode of the primary cavity, exceeding its losses, which then starts to appear in the P1 output.

For increasing AOM diffraction loss, the primary laser output intensity profile was smoothly transformed from the donut-shaped distribution, i.e. $\mathrm{LG}_{01}$ mode, to a shallow crater-shaped distribution, and finally, to a quasi-top-hat distribution. The latter became when the additional round-trip loss reached $\sim 2.5 \%$, for the pump power around $3.2 \mathrm{~W}$. Secondary-cavity-losses and the cavity-photon-density of the lasing mode(s) define the population inversion distribution in the

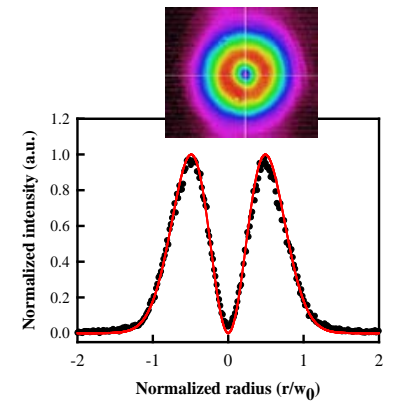

(a)

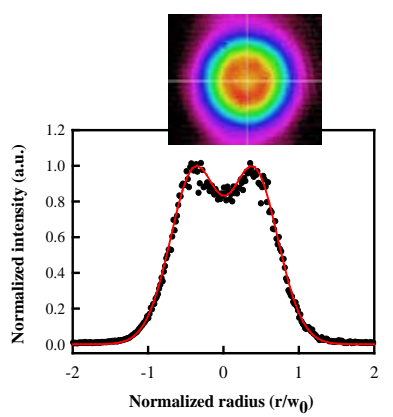

(c)

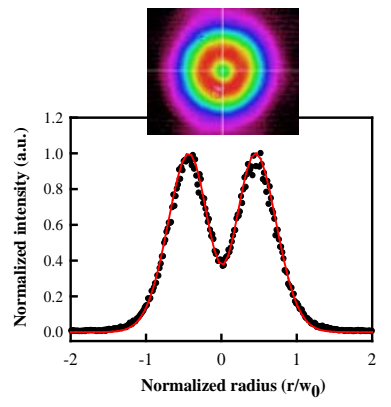

(b)

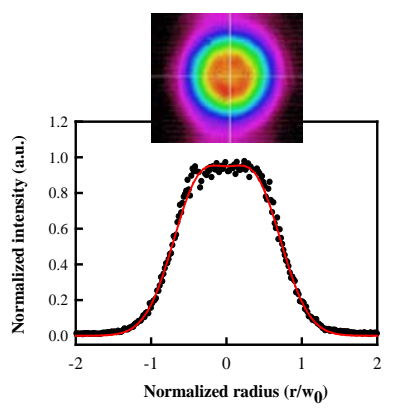

(d)
Fig. 2. Beam profiles and measured transverse intensity distributions of the laser output from the primary cavity, at the incident pump power of $3.2 \mathrm{~W}$, for the additional AOM diffraction losses of (a) $0 \%$, (b) $0.5 \%$, (c) $1.5 \%$, and (d) $2.5 \%$. The red lines are the theoretical intensity distributions calculated with Eq. (1) and $\gamma=0,0.19,0.5$ and 0.82 respectively. 
Nd:YAG crystal [18]. Therefore, increasing losses in the secondary cavity, operating on its fundamental mode, forces a buildup in the available gain in the central volume of the pumped region. This region has the highest overlap with the TEM 00 mode of both cavities. As the two cavities are decoupled by the polarization selective element, the corresponding gain distribution in the common gain element surpasses the $\mathrm{TEM}_{00}$ mode threshold condition for lasing in the primary cavity, therefore contributing to the P1 output and "filling in" the central "hole" of the $\mathrm{LG}_{01}$ mode. While the threshold pump power and the slope efficiency for the TEM 00 mode in the primary cavity depend on the lasing state of the secondary cavity, lasing performance for the $\mathrm{LG}_{01}$ mode is not. Since the latter mode was suppressed in the secondary cavity by AP, the gain distribution of the outer annulus of the pumped region is defined by the primary cavity losses. A theoretical model for this mechanism is being developed, but is beyond the scope of this paper.

The laser output power as a function of incident pump power was measured for the different AOM losses of $0 \%, 0.5 \%, 1.5 \%$ and $2.5 \%$, as seen in Fig. 3. We monitored the stability in the output power and the beam profile and found them to be within the usual bounds of the single linear cavity design used. It should be noted that the Nd:YAG cavities were designed and configured to produce an optimized overlap of the respective cavity modes in the gain crystal to ensure tunable control of the beam profile with the AOM losses trialed. The maximum output powers achieved were $390 \mathrm{~mW}, 410 \mathrm{~mW}, 460 \mathrm{~mW}$ and $510 \mathrm{~mW}$ corresponding to the slope efficiency of $28.8 \%, 26.5 \%$, $26.4 \%$ and $32.1 \%$ for the AOM losses of $0 \%, 0.5 \%, 1.5 \%$ and $2.5 \%$ respectively. Despite a linear increase of the output power, the shape of the output beam profile was not maintained with increasing pump powers. Figure 4 shows the output beam profiles at the different power levels for the additional AOM losses of $0 \%, 0.5 \%$ and $1.5 \%$, respectively. It is clearly seen that the output beam profile was dependent upon the power level, except for the case when there was no additional AOM loss. Assuming that the output was an incoherent superposition of the TEM 00 and $\mathrm{LG}_{01}$ modes, we compared the measured beam profiles with the following equation for the theoretical transverse intensity distribution:

$$
I_{\text {top-hat }}(r)=\gamma I_{0,0}(r)+I_{0,1}(r)=\left[\gamma+\left(\frac{2 r^{2}}{w^{2}}\right)\right] I_{0,0}(r)
$$

where $\mathrm{I}_{0,0}(\mathrm{r})$ and $\mathrm{I}_{0,1}(\mathrm{r})$ are the normalized intensity profiles for the $\mathrm{TEM}_{00}$ and $\mathrm{LG}_{01}$ modes respectively, $\gamma$ is a scaling factor for the relative intensity of the TEM 00 mode to the $\mathrm{LG}_{01}$ mode, and, $w$ is the

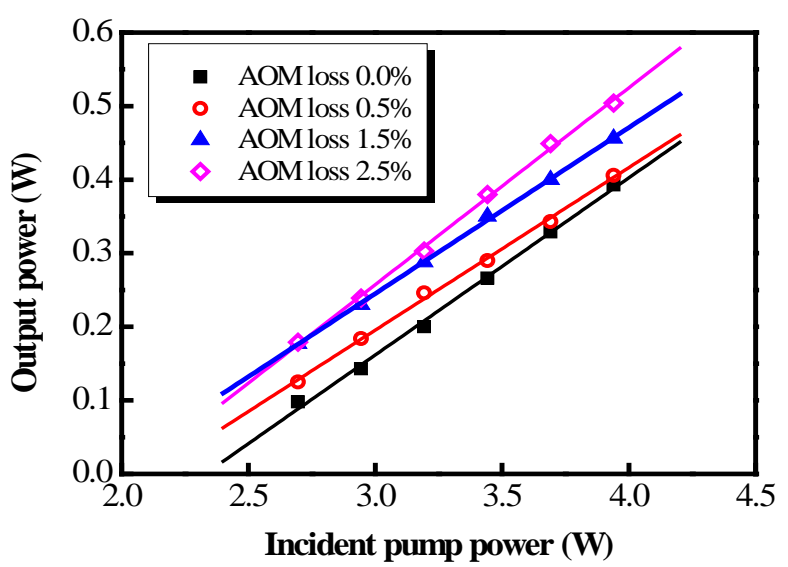

Fig. 3. Nd:YAG laser output power (P1) as a function of incident pump power for the additional AOM diffraction losses of $0 \%, 0.5 \%, 1.5 \%$, and $2.5 \%$. waist for the constituent TEM $_{00}$ beam. Theoretical calculations using Eq. (1) show that the combined intensity distribution is closest to a tophat shape for $\gamma=1$, as seen in Fig. 5(a). We empirically determined the magnitude of the scaling factor $\gamma$ for the various experimental conditions tested, fitting Eq. (1) to the measured near-field beam profiles. Fig. 5(b) shows the result as a function of the pump power for different AOM losses. As the pump power was increased, it was observed that $\gamma$ decreased and converged to a value less than 1 , resulting in a crater-shaped beam profile at the highest pump powers trialed. This effect on the primary cavity output beam is a result of the higher slope efficiency for the $\mathrm{LG}_{01}$ mode compared with the TEM 00 mode. When we suppressed lasing in the secondary cavity, by closing the AP completely, we could obtain a quasi-top-hat beam with $\gamma \approx 1.0$. This demonstrates that the scaling factor, thus beam profile shape, is dependent on the TEM 00 lasing condition of the secondary cavity, which extracts the stored energy from the central volume of the pumped region in the laser medium. Moreover, the pump beam volume had been carefully chosen to provide an optimal overlap for the first two modes of the primary cavity [16]. As such the relative contribution of each mode of the isolated primary cavity was nominally equal, i.e. $\gamma \approx 1$. A larger pump spot would favor a smaller $\gamma$, with a greater contribution coming from the $\mathrm{LG}_{01}$ mode but potentially allow for $\mathrm{LG}_{02}$ to reach threshold, which is not accounted for in this simple scaling factor. Alternatively, a smaller pump beam, favoring mode matching with the fundamental cavity mode, could increase $\gamma$, as is the norm for end-pumped solid-state lasers, which generally target $\gamma \approx \infty$.

Evidently, from the results presented herein, employing the dualcavity configuration enables the tailored beam profile from the primary cavity, via adjustment of the cavity loss in the secondary cavity. The AOM in the secondary cavity could in fact be replaced with another optical device, such as an electro-optic modulator, or an angled etalon, which also provides precise control of intra-cavity loss. That is to say, the method is not limited to use of an AOM, but will be transferrable to any suitable method of adjusting the cavity- $Q$ dynamically.
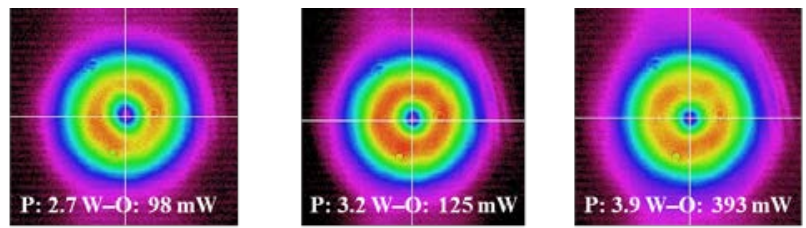

(a)
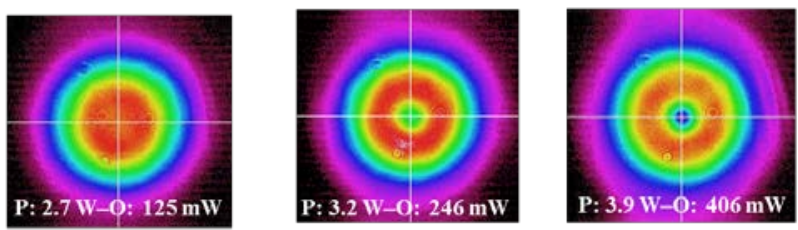

(b)
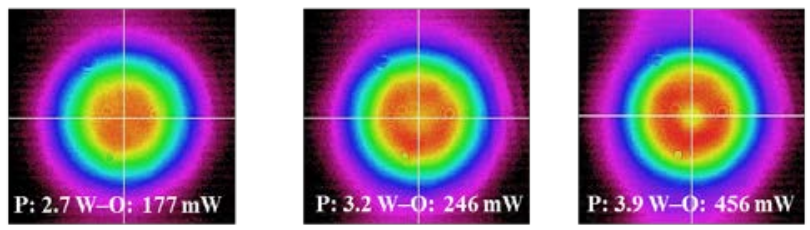

(c)

Fig. 4. Output beam profiles at an incident pump power (P) of $2.7 \mathrm{~W}, 3.2 \mathrm{~W}$, and $3.9 \mathrm{~W}$ for the AOM losses of (a) $0 \%$, (b) $0.5 \%$, and (c) $1.5 \%$. 


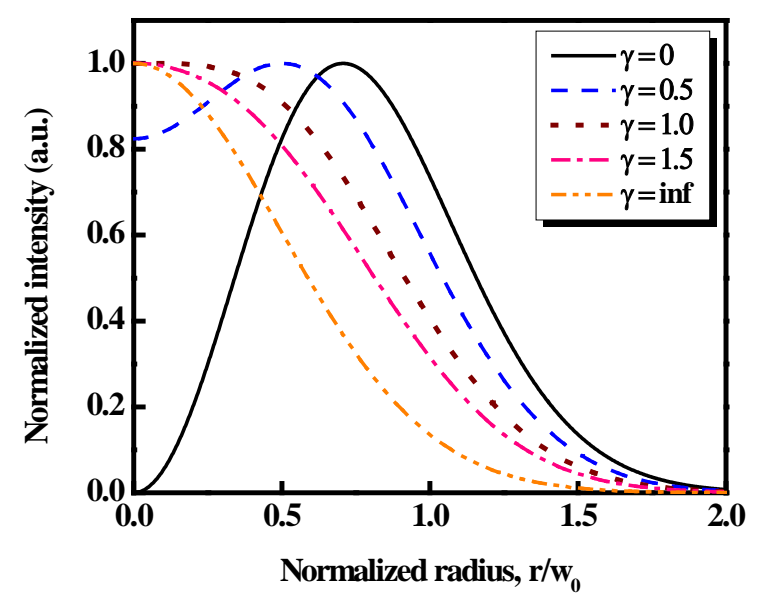

(a)

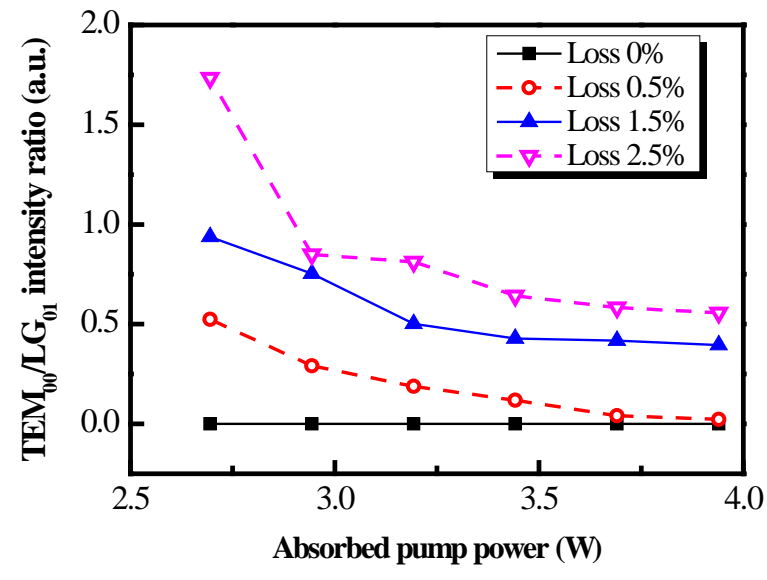

(b)

Fig. 5. (a) Calculated intensity distributions of laser beams (P1) with different scaling factors, $\gamma$, from Eq. (1), and, (b) the fitted scaling factor $\gamma$ as a function of incident pump power for different AOM losses. inf : infinite

Furthermore, the dual-cavity configuration will also allow laser operation in a Q-switched mode, similar to that reported by Arbabzadah et al. [19], by adding another Q-switch in the primary cavity, benefitting from higher damage threshold limits, better extraction efficiency, and being able to tailor the resultant beam profile dynamically.

In summary, we have demonstrated a novel technique for direct generation of tailored beam profiles using a dual-cavity laser configuration. This technique has been applied to a Nd:YAG laser with a controllable AOM diffraction loss in a secondary cavity, used to define the appropriate gain distribution in the primary cavity, and thus produce spatial intensity distributions ranging from a pure donutbeam to one with a quasi-top-hat profile. In addition, we analyzed the ratio of the respective contributions of the $\mathrm{TEM}_{00}$ mode to the $\mathrm{LG}_{01}$ mode in the output beam, as a function of the AOM loss. With this knowledge, we were able to define the optimum conditions for generating a desired beam profile, which could be changed rapidly via control of the AOM diffraction efficiency. This approach will be advantageous for adaptable beam profiles in high-power solid state laser systems, with excellent potential for Master Oscillator Power Amplifier systems and Q-switched modes of operation. Furthermore, this method provides very good extraction efficiency of the stored energy, coupled with the potential for higher pulse energy extraction before damage threshold limits are reached. This is primarily due to better amplification uniformity across the oscillating modes, by controlling the gain distribution profile via the operating conditions of the secondary cavity. We believe the simplicity and flexibility afforded by this technique will benefit a range of applications requiring laser beams with high power and a bespoke intensity profile.

Funding. National Research Foundation of Korea (NRF); Basic Science Research Program (NRF-2014R1A1A2A16053885)

\section{References}

1. M. R. E. Lamont, Y. Okawachi, and A. L. Gaeta, Opt. Lett. 38, 3478 (2013). W. Koechner, Solid State Laser Engineering (Springer-Verlag, 2006), 407418.

2. O. Homburg, F. Völkermeyer, F. Toennissen, H. Ganser, and T. Mitra, Proceedings of WLT Conference on Lasers in Manufacturing (Munich, Germany, 2007).

3. E. Wyss, M. Roth, T. Graf, and H. Weber, IEEE J. Quantum Electron. 38, 1620-1628 (2002).

4. L. A. Abramyan and G.A. Pasmanik, Sov. J. Quantum Electron. 20, 11261128 (1990).

5. C. Valentin, P. Calvet, Y. Quiquempois, G. Bouwmans, L. Bigot, Q. Coulombier, M. Douay, K. Delplace, A. Mussot, and E. Hugonnot, Opt. Express 21, 23250-23260 (2013).

6. Y. Matsuura, D. Akiyama, and M. Miyagi, App. Opt. 42, 3505-3508 (2003).

7. F. Wippermann, U. Zeitner, P. Dannberg, A. Brauer, and S. Sinzinger, Opt. Express 15, $6218-6231$ (2007).

8. D. L. Shealy and S. H. Chao, Opt. Eng. 42, 3123-3138 (2003).

9. V. Bagnoud and J. D. Zuegel, Opt. Lett. 29, 295-297 (2004).

10. A. J. Caley, M. J. Thomson, J. Liu, A. J. Waddie, and M. R. Taghizadeh, Opt. Express 15, 10699-10704 (2007).

11. P. Bélanger and C. Paré, Opt. Lett. 16, 1057-1059 (1991)

12. M. Gerber and T. Graf, IEEE J. Quantum Elect. 40, 741-746 (2004).

13. J. W. Kim, J. I. Mackenzie, J. R. Hayes, and W. A. Clarkson, Opt. Lett. 37, 1463-1465 (2012).

14. T. Dietrich, M. Rumpel, T. Graf, and M. A. Ahmed, Optics Express 23, 26651-26659 (2015).

15. D. J. Kim and J. W. Kim, Appl. Phys. B 121, 401-405 (2015).

16. J. W. Kim and W. A. Clarkson, Opt. Commun. 296, 109-112 (2013).

17. W. Koechner, Solid State Laser Engineering (Springer-Verlag, 2006), 123125.

18. K. Kubodera, and K. Otsuka, J. Appl. Phys. 50, 653-659 (1979).

19. E. A. Arbabzadah, P. C. Shardlow, A. Minassian, and M. J. Damzen, Opt. Lett. 39, 3437-3440 (2014). 


\section{Full references}

1. W. Koechner, Solid State Laser Engineering (Springer-Verlag, 2006), 407-418.

2. O. Homburg, F. Völkermeyer, F. Toennissen, H. Ganser, and T. Mitra, "High-precision Gaussian-to-tophat beam transformation improves structure quality and speed in micro-machining," Proceedings of WLT Conference on Lasers in Manufacturing (Munich, Germany, 2007).

3. E. Wyss, M. Roth, T. Graf, and H. Weber, "Thermooptical compensation methods for high-power lasers,” IEEE J. Quantum Electron. 38, 1620-1628 (2002).

4. L. A. Abramyan and G.A. Pasmanik, "Deformation of the laser beam profile as a result of thermal defocusing and its Influence on the stimulated Brillouin scattering threshold for long pulses," Sov. J. Quantum Electron. 20, 1126-1128 (1990).

5. C. Valentin, P. Calvet, Y. Quiquempois, G. Bouwmans, L. Bigot, Q. Coulombier, M. Douay, K. Delplace, A. Mussot, and E. Hugonnot, "Top-hat beam output of a single-mode microstructured optical fiber: Impact of core index depression,” Opt. Express 21, 23250-23260 (2013).

6. Y. Matsuura, D. Akiyama, and M. Miyagi, "Beam homogenizer for hollow-fiber delivery system of excimer laser light,” App. Opt. 42, 3505-3508 (2003).

7. F. Wippermann, U. Zeitner, P. Dannberg, A. Brauer, and S. Sinzinger, "Beam homogenizers based on chirped microlens arrays,” Opt. Express 15, 6218 -6231 (2007).

8. D. L. Shealy and S. H. Chao, "Geometric optics-based design of laser beam shaper,” Opt. Eng. 42, 3123-3138 (2003).

9. V. Bagnoud and J. D. Zuegel, "Independent phase and amplitude control of a laser beam by use of a single-phase-only spatial light modulator,” Opt. Lett. 29, 295-297 (2004).

10. A. J. Caley, M. J. Thomson, J. Liu, A. J. Waddie, and M. R. Taghizadeh, "Diffractive optical elements for high gain lasers with arbitrary output beam profiles,” Opt. Express 15, 1069910704 (2007).

11. P. Bélanger and C. Paré, "Optical resonators using gradedphase mirrors,” Opt. Lett. 16, 1057-1059 (1991)

12. M. Gerber and T. Graf, "Generation of super-Gaussian modes in Nd:YAG lasers with a graded-phase mirror," IEEE J. Quantum Elect. 40, 741-746 (2004).

13. J. W. Kim, J. I. Mackenzie, J. R. Hayes, and W. A. Clarkson, "High-power Er:YAG laser with quasi-top-hat output beam," Opt. Lett. 37, 1463-1465 (2012).

14. T. Dietrich, M. Rumpel, T. Graf, and M. A. Ahmed, "Investigations on ring-shaped pumping distributions for the generation of beams with radial polarization in an Yb:YAG thindisk laser," Optics Express 23, 26651-26659 (2015).

15. D. J. Kim and J. W. Kim, "High-power TEM00 and LaguerreGaussian mode generation in double resonator configuration,” Appl. Phys. B 121, 401-405 (2015).

16. J. W. Kim and W. A. Clarkson, "Selective generation of Laguerre-Gaussian( $\mathrm{LG}_{0 \mathrm{n}}$ ) mode output in a diode-laser pumped Nd:YAG laser,” Opt. Commun. 296, 109-112 (2013).

17. W. Koechner, Solid State Laser Engineering (Springer-Verlag, 2006), 123-125.

18. K. Kubodera, and K. Otsuka, "Single-transverse-mode $\mathrm{LiNdP}_{4} \mathrm{O}_{12}$ slab waveguide laser,” J. Appl. Phys. 50, 653-659 (1979).

19. E. A. Arbabzadah, P. C. Shardlow, A. Minassian, and M. J. Damzen, "Pulse control in a Q-switched $\mathrm{Nd}: \mathrm{YVO}_{4}$ bounce geometry laser using a secondary cavity,” Opt. Lett. 39, 34373440 (2014). 